\title{
Sensor-Fused Autonomous Guidance of a Mobile Robot and Applications to Mars Sample Return Operations
}

\author{
T. L. Huntsberger ${ }^{a}$, E. T. Baumgartner ${ }^{a}$, H. Aghazarian ${ }^{a}$, Y. Cheng ${ }^{a}$, P. S. Schenker ${ }^{a}$ \\ P. C. Leger ${ }^{b}$, K. D. Iagnemma ${ }^{c}$, and S. Dubowsky ${ }^{c}$ \\ ${ }^{a}$ Science and Technology Development Section, Jet Propulsion Laboratory, \\ California Institute of Technology, Pasadena, CA 91109 \\ ${ }^{b}$ Robotics Institute, Carnegie Mellon University, \\ Pittsburgh, PA 15213 \\ ${ }^{c}$ Department of Mechanical Engineering, Massachusetts Institute of Technology, \\ Cambridge, MA 02215
}

\begin{abstract}
Generally, there are multiple sensor suites on existing rover platforms such as NASA's Sample Return Rover (SRR) and the Field Integrated Design and Operations (FIDO) rover at JPL. Traditionally, these sensor suites have been used in isolation for such tasks as planetary surface traversal. For example, although distant obstacle information is known from the narrow FOV navigation camera (NAVCAM) suite on SRR or FIDO, it is not explicitly used at this time for augmentation of the wide FOV hazard camera (HAZCAM) information for obstacle avoidance. This paper describes the development of advanced rover navigation techniques. These techniques include an algorithm for the generation of range maps using the fusion of information from the NAVCAMs and HAZCAMs, and an algorithm for registering range maps to an a priori model-based range map for relative rover position and orientation determination. Experimental results for each of these techniques are documented in this paper.
\end{abstract}

Keywords: Rover navigation, state estimation, map registration

\section{INTRODUCTION}

As part of the NASA Telerobotic (TR) and Exploration Technology (ET) programs, the Jet Propulsion Laboratory (JPL) has recently developed two prototype rovers, SRR and FIDO, both shown in Figure 1. Current baseline plans for the 2003/2005 Mars Sample Return (MSR) missions include long range surface traverses for the collection of core samples acquired with an onboard mini-corer drill. These samples are then returned to the lander that would launch them into Mars orbit via a Mars Ascent Vehicle for eventual return to Earth. Figure 2 is an illustration of a possible set of operations for the cache return to the lander. Since the time for sample collection is currently set at 90 sols, there will be a need for efficient navigation algorithms for long range traverses coupled with autonomous rover localization techniques for both far field $(125 \mathrm{~m})$ as well as close $(1 \mathrm{~m})$ approaches in order to successfully complete the mission requirements.

The sensing and computing architectures are very similar in the two rovers. Both rovers have forward facing stereo camera pair (HAZCAMs) for hazard avoidance and a mast mounted stereo camera pair (NAVCAMs) for long-range sensing. The main difference is in the mast height above the ground, which is $93 \mathrm{~cm}$ for SRR and $1.94 \mathrm{~m}$ for FIDO. The same computing architecture (PC104+ stack with 300 Mhz AMD K6 CPU and 16 MB RAM)) is used on both platforms. These similarities simplify the cross-development of algorithms for the two rover platforms.

A major difference between the rovers lies in the mobility sub-systems. Whereas SRR is a four-wheeled rover platform with independent steering on all wheels and a split differential and independently-controlled shoulder (or rocker) joints, FIDO has a six-wheel, independently-steered rocker-bogie mobility configuration which is geometrically scaled by a factor of 20/13 in proportion to the analogous Mars Pathfinder Sojourner rover configuration. In addition, SRR is a $7+$ kilogram class rover for fast, short range traverses, while FIDO is a $60+$ kilogram class rover for multikilometer traverses, carrying a set of five scientific spectrometers and a robotic mini-coring and caching system.

Send correspondence to T. L. Huntsberger, E-mail: Terry.Huntsberger@jpl.nasa.gov. 


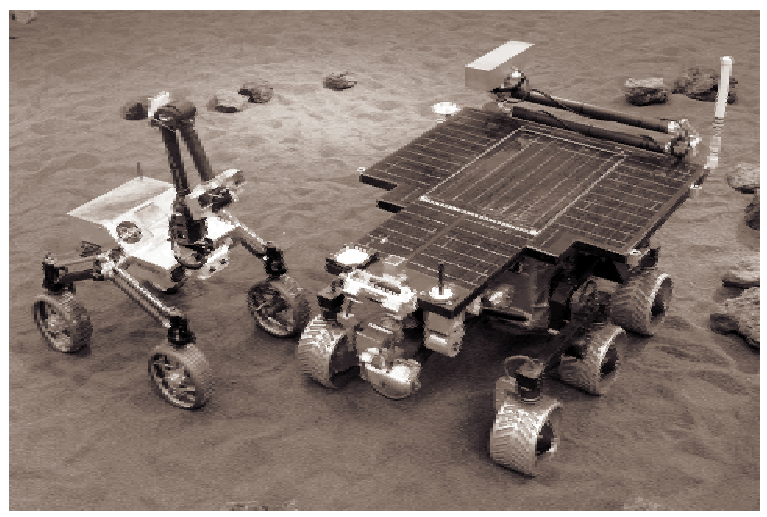

Figure 1. NASA's SRR and FIDO in the Planetary Robotics Lab at JPL.

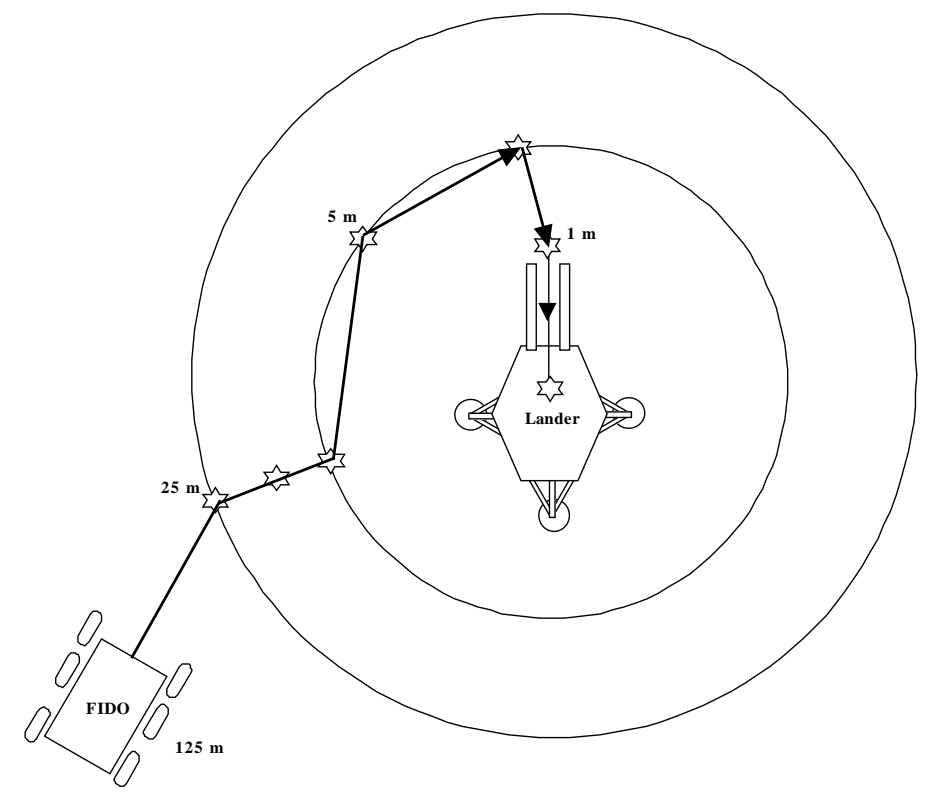

Figure 2. Possible sequence of operations for sample cache return to lander during 2003/2005 missions (drawing not to scale).

We have developed a number of different navigation techniques and algorithms for the long range traversals far from the lander and for the rendezvous/docking with the lander for the sample cache hand-off to the MAV. These include visual localization techniques for finding the rover in the mast camera's field-of-view, visual guidance techniques for approaching the lander from far distances in rough uneven terrain, and visual terminal guidance techniques for rover localization and alignment with the ramps prior to the ascent to the lander deck. Some of these methods were described in the paper at this conference last year. ${ }^{1}$ We will concentrate in this paper on the two technology needs, visual guidance for long range rover navigation and visual terminal guidance for the final lander/ramp approach.

Section 2 describes recent developments and preliminary experimental trials for a stereo multimap fusion algorithm that provides an added measure of rover safety for long range traverses in the presence of obstacles. The adaptation 
of the map registration technique reported last year to the accurate terminal positioning of a rover with respect to the lander ramps is presented in Section 3. Finally, some concluding remarks are presented in Section 4.

\section{MULTIMAP FUSION FOR ROVER NAVIGATION}

The range maps that are derived from the rover HAZCAMs are typically sparse ( $50 \%$ missing values) due to lens distortions, poor contrast from extreme lighting conditions, and shadow regions behind obstacles. Navigation within the bounds of these missing points discounts potential paths toward a goal, since these areas in the range maps are off limits in order to maintain rover safety. In addition, the total loss of HAZCAMs as occurred during a recent Mars Yard trial severely hampers the rover from goal achievement. With the current short mission duration missions and power constraints for rovers on planetary surfaces, the loss of these paths can lead to mission failure.

The use of multiple range maps taken by different sensors for improved rover localization in rough, uneven terrain was recently investigated by Olson and Matthies, et al. ${ }^{2-5}$ They used a Hausdorff metric and an optimized iconic matching algorithm. ${ }^{6}$ Experiments with the Rocky7 rover in the Mars Yard at JPL indicated that localization to within 1 to $2 \mathrm{~cm}$ was possible with a modest computational investment within the bounds of rover constraints.

The current obstacle avoidance/local navigation algorithm used on FIDO recently was field tested in the Mojave Desert at Silver Lake, CA in April of 1999. Long range traversals of 75 to 100 meters based on dead reckoning resulted in a localization error of only $3 \%$ as ground truthed using GPS. The algorithm uses a series of decision-making steps with local range maps derived from the HAZCAMs to move in increments of 2 meter traverses. It is anticipated that the use of a fast stereo algorithm developed at JPL will allow continuous movement. A history of rover state and previous range maps is kept for back-tracking in case of rover entrapment by obstacles. The algorithm is goal-biased which can lead to non-optimal behavior since it is only using the local range maps for path analysis.

An extension to this algorithm makes use of the NAVCAMs for a multimap path analysis. This is accomplished with a 3 phase algorithm, the steps of which are:

- Determine necessary rover heading to goal and check for obstacles in the local range maps if turn is made in that direction. An analysis of potential straight line paths is made during this phase (path consists of straight line motion and turn-in-place). Full rover size, mobility, and clearance constraints are used in this calculation.

- If the direct path is blocked, servo the mast to acquire a series of NAVCAM wedges in the goal direction that will reveal other potential paths and fill in missing range data. Since the rover has not moved yet, range map registration is easily accomplished using the mast/rover kinematic relations. Potential paths are evaluated using the same method as in the first phase.

- Start to traverse along the path returned during phase 1 or phase 2, using the local range maps for fine adjustments. Since the rover has now moved, wheel odometry can be used to match into the NAVCAM acquired range maps although the wheel data tends to be very unreliable due to slippage. Incorporation of physics-based rover planning with full kinematics and sensor modeling can improve this process. ${ }^{7}$ In addition, recent work reported at this conference last year demonstrated substantial improvement in rover localization using an extended Kalman filter approach to fusing visual and odometry data. ${ }^{1,8}$

- As the goal is approached, take NAVCAM wedges to completely cover the area around the goal for further planning.

This method was tested in the Mars Yard at JPL using data derived from the HAZCAMs and NAVCAMs on FIDO. A NAVCAM panorama of $135 \times 55$ degrees is shown in Figure 3. FIDO was centered at the bottom of the frame. The HAZCAM stereo pair and derived range map are shown in Figures 4 and 5 respectively. There was a 25 $\mathrm{cm}$ obstacle directly in front of the rover, and the goal was approximately in the center of the NAVCAM panorama. FIDO made the correct decision to turn right to avoid the obstacle, leading to a path that diverged from a direct one to the goal. A problem with the HAZCAMs led to poor range maps (100\% no range) for the next two steps, so the path continued to diverge from the goal. This led to a final localization error relative to the goal of 3.5 meters which was totally attributable to the loss of the HAZCAMs.

Examination of the previously taken NAVCAM panorama data indicates that a relatively open path exists to the goal once the initial obstacle was avoided. Post-trial simulation with the 3 phase algorithm discussed above using 
range maps derived from this data yielded a localization error relative to the goal of $25 \mathrm{~cm}$. Wheel slippage for rover turn-in-place was set to $4 \%$ and during traverses was set to $12 \%$ based on data seen during this and previous Mars Yard experiments.

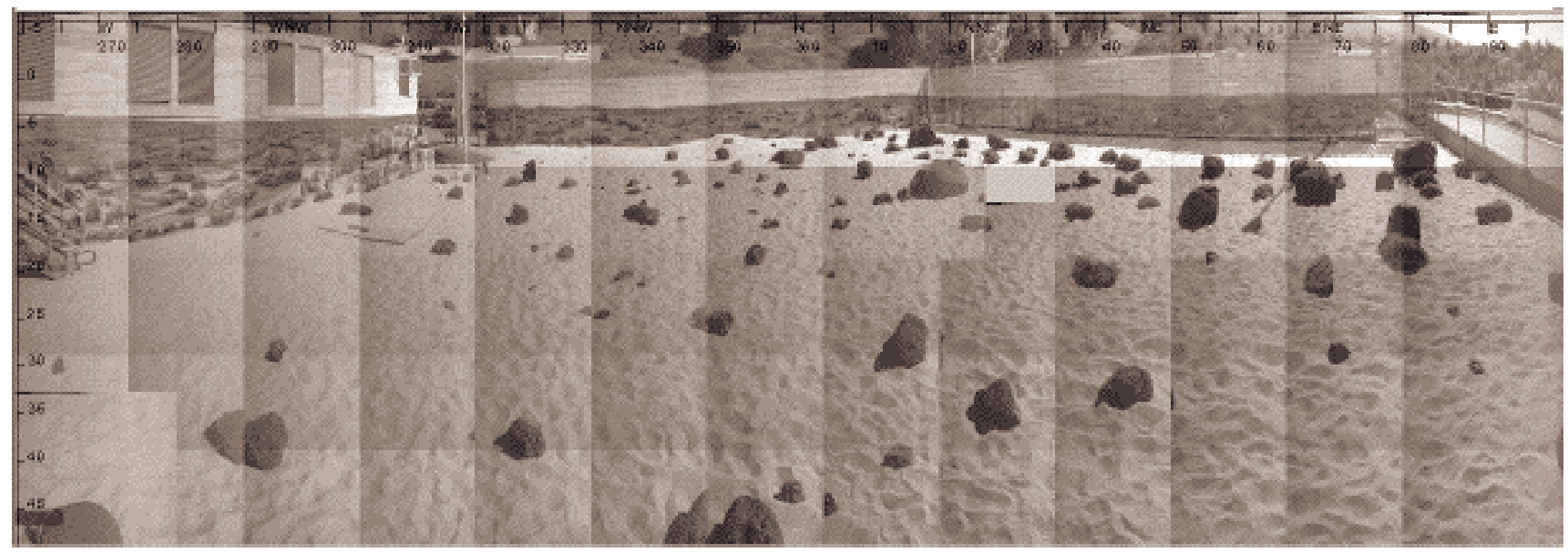

Figure 3. Reconstructed panorama (135X55 degrees) taken by FIDO NAVCAMs ( $25 \mathrm{~cm}$ baseline) in Mars Yard at JPL.
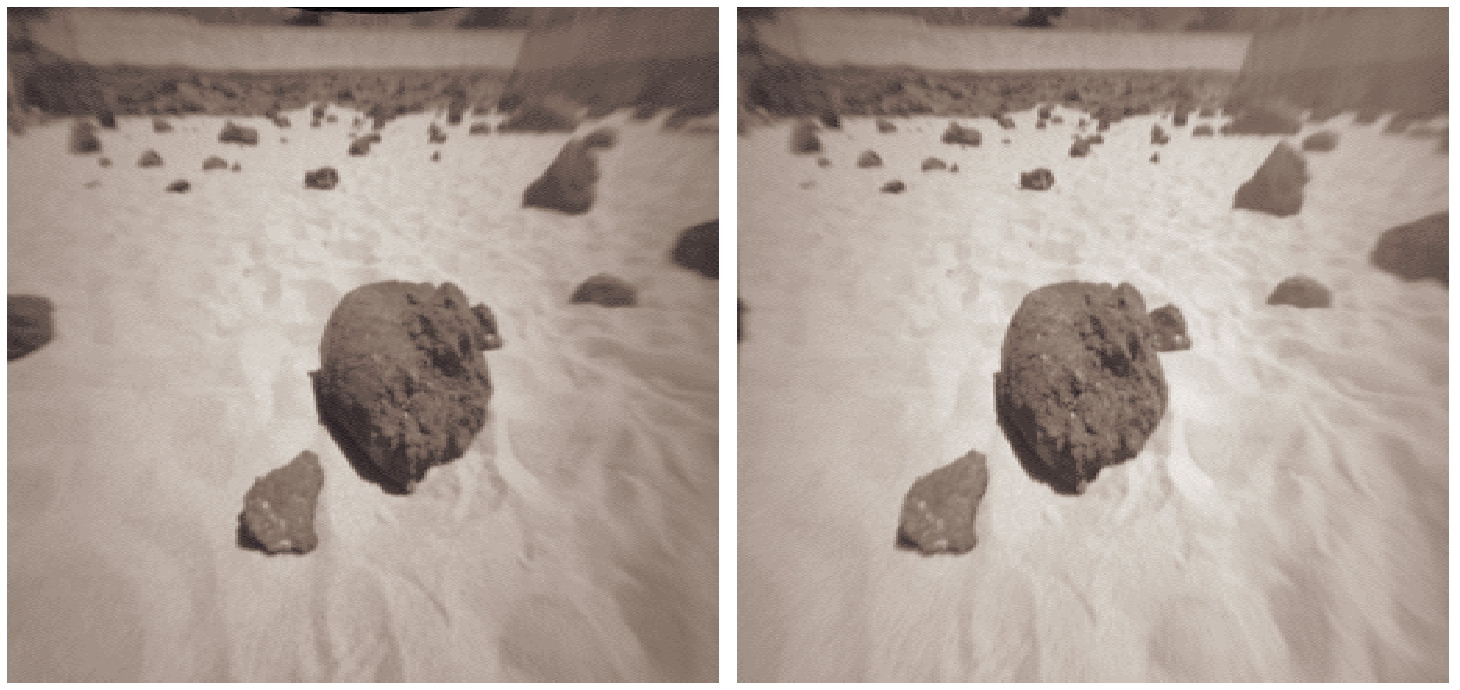

Figure 4. Stereo pair from FIDO HAZCAMS (10 $\mathrm{cm}$ baseline) with heading centered in Figure 3 .

\section{GOAL LOCALIZATION}

Rover misalignment during the final approach to the ramps before ascent onto the lander deck is a potential single point failure for the 2003/2005 MSR missions. Tests undertaken in the Planetary Robotics Lab at JPL have demonstrated that rover misalignment to the ramps is limited to $1.5 \mathrm{~cm}$ in lateral offset. Beyond this bound, the rover tends to drive over the side of the guide rails on the ramps. Results presented at this meeting last year demonstrated rover self-localization and relative pose estimate of a target science rover during final approach well within this bound. ${ }^{1}$ 


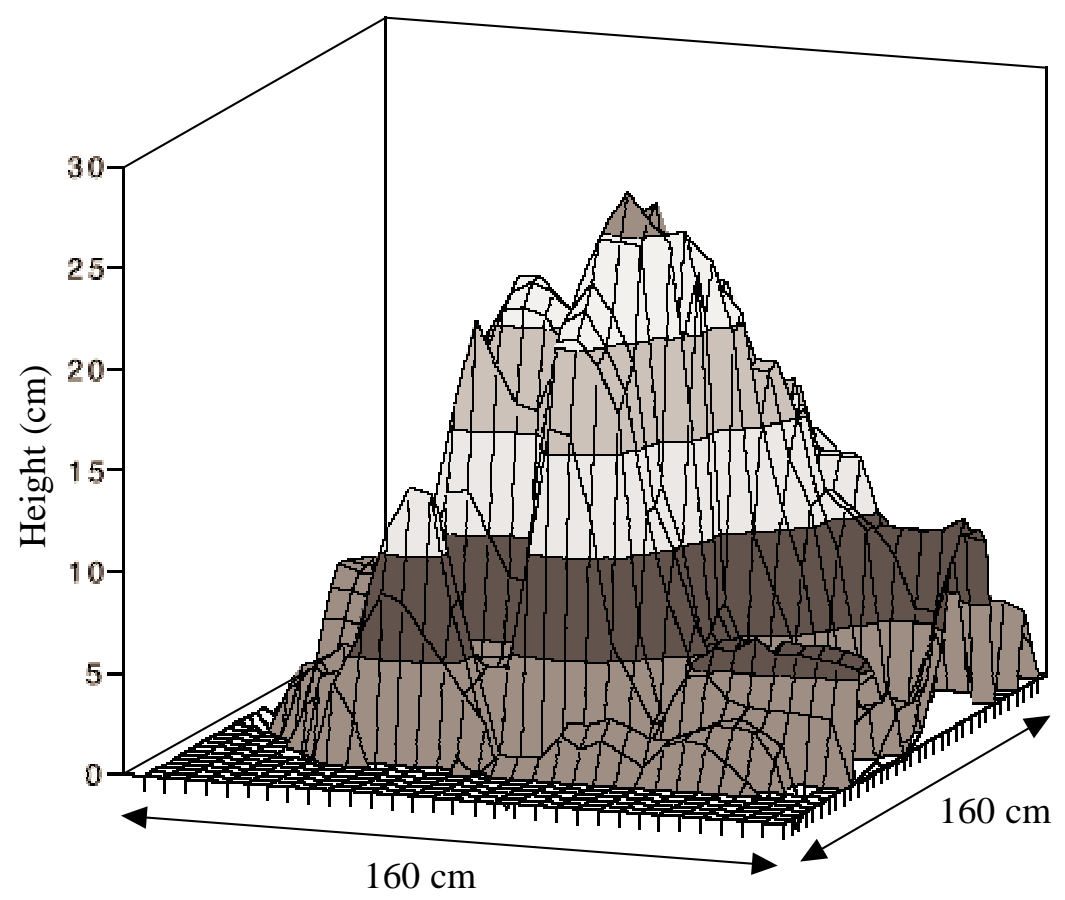

Figure 5. Local heightfield derived from stereo pair of Figure 4.

$3 \mathrm{D}$ registration of range maps acquired during the terminal approach to a priori models of the goal taken at various standoff distances was used to successfully retrieve a cache container from a static science rover.

The technique matches into a $\mathrm{k}$-D tree representation of the range models using a closest point algorithm ${ }^{9}$ This is followed by the application of a dual number quaternion method ${ }^{10}$ that computes a rotation matrix and translation vector which minimize the sum of the squared distances between corresponding points. Motion between poses is estimated based on a comparison of the transformations generated for two nearby rover positions. Rover motion in the far field ( $1 \mathrm{~m}$ standoff) tend to be on the order of 50-60 cm, in mid-range (50 cm standoff) on the order of 10 $\mathrm{cm}$, and in the final approach on the order of $2 \mathrm{~cm}$. Heading errors due to slippage during turns is mitigated through the acquisition of stereo maps after each move.

This technique was applied to the ramp localization problem using SRR and a mockup of the 2003/2005 lander shown in Figure 6. An example of registration between a ramp range map and a map acquired at a $30 \mathrm{~cm}$ standoff location is shown in Figure 7. A series of 15 trials using SRR and the lander mockup were run in the Planetary Robotics Lab at JPL. The average lateral offset for these trials was $2.6 \mathrm{~cm}$, and the average goal distance error was $4.7 \mathrm{~cm}$ with an average heading error of 1.2 degrees. The error in the lateral offset can be traced to the relatively sparse range maps of the lander ramps. The goal distance error can be traced to the resolution of the range maps where relative distance errors of $10 \%$ are not uncommon. The contrast necessary for a dense range map based on stereo correlation is not present in the ramps, with good data generally being found in the edges of patterns that were applied to the ramps (see Figures 6 and 7 ). Current mission baseline for the lander ramp design includes a 5 degree funnel in the guide rails at the base of the ramps, which increases the lateral offset error bound to $4 \mathrm{~cm}$. This indicates that our technique will be able to perform within this error tolerance.

\section{CONCLUSIONS}

We have presented rover technology developments for safer long range navigation and accurate lander ramp localization for cache hand-off. A simulation of the 3 phase multimap fusion algorithm using data derived from the HAZCAM and NAVCAM sensors of FIDO successfully solved the problem of loss of a single sensing modality (HAZCAMs) for a traverse to a known goal location. We also demonstrated the application of a map registration technique to the lander ramp localization task for the 2003/2005 MSR missions. The errors in goal localization and ramp alignment by the rover were well within tolerances established for mission baselines. Inclusion of the EKF fused visual/odometry method detailed in Hoffman, et $\mathrm{al}^{8}$ should further improve the distance to goal errors encountered during both of 


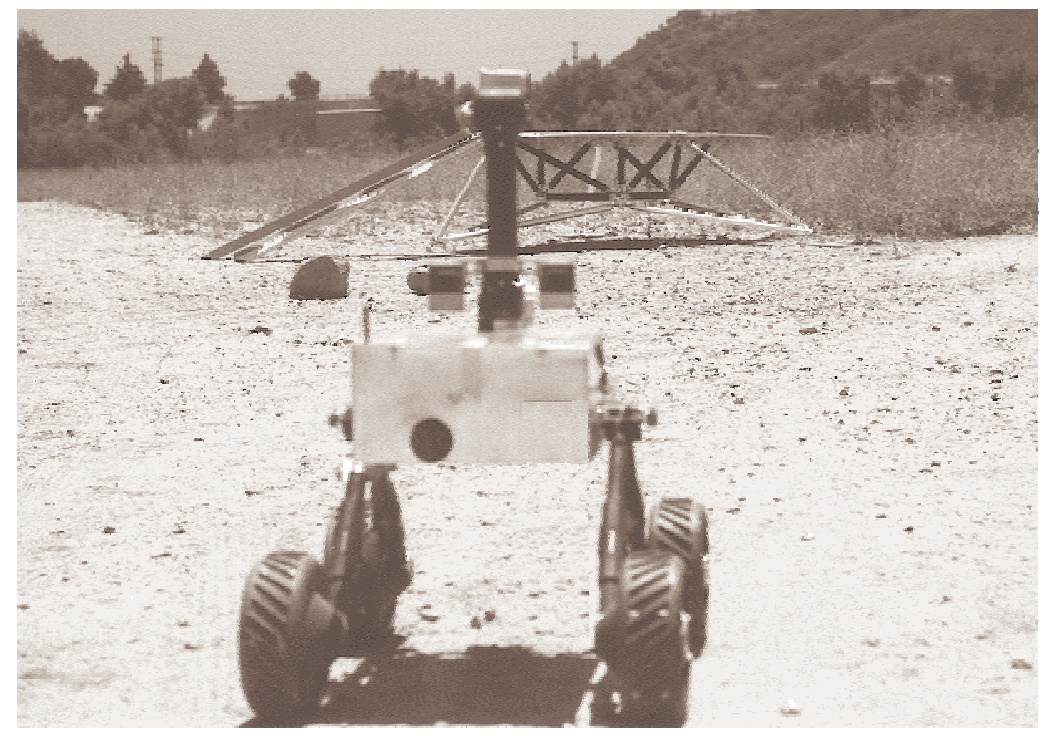

Figure 6. SRR and 2003/2005 lander mockup in arroyo at JPL.

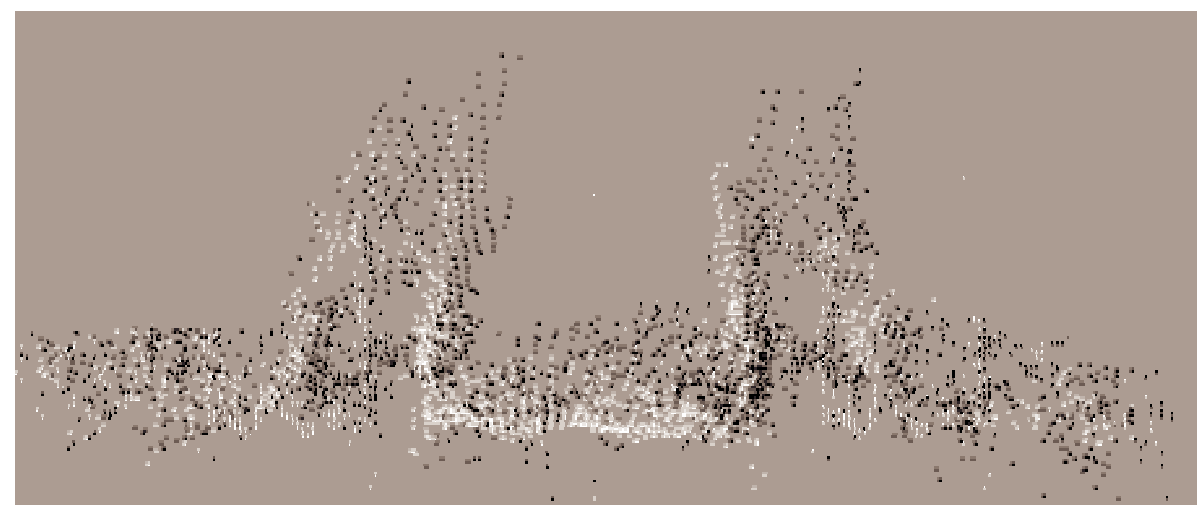

Figure 7. Registration of model (black) to the scene data (white), which was acquired at distance of approximately $30 \mathrm{~cm}$ from the lander ramps.

these tasks. We are also investigating the incorporation of the physics-based modeling of rover/terrain interactions into the path analysis process. ${ }^{7}$

\section{ACKNOWLEDGMENTS}

The research described in this paper was carried out by the Jet Propulsion Laboratory, California Institute of Technology, under a contract with the National Aeronautics and Space Administration. 


\section{REFERENCES}

1. E. T. Baumgartner, P. C. Leger, P. S. Schenker, and T. L. Huntsberger, "Sensor-fused navigation and manipulation from a planetary rover," in Proc. SPIE, vol. 3523, pp. 58-66, 1998.

2. L. H. Matthies, C. F. Olson, G. Tharp, and S. Laubach, "Visual localization methods for Mars rovers using lander, rover, and descent imagery," in 4th International Symposium on Artificial Intelligence, Robotics and Automation in Space, pp. 413-418, 1997.

3. C. F. Olson, "Mobile robot self-localization by iconic matching of range maps," in 8th International Conference on Advanced Robotics, pp. 447-452, 1997.

4. C. F. Olson and L. H. Matthies, "Maximum Likelihood rover localization by matching range maps," in IEEE ICRA 98, pp. 272-277, 1998 .

5. C. F. Olson, "Subpixel localization and uncertainty estimation using occupancy grids," in IEEE ICRA 99, pp. 1987-1992, (Detroit, MI), May 1999.

6. C. F. Olson and D. P. Huttenlocher, "Automatic target recognition by matching oriented edge pixels," IEEE Transactions on Image Processing 6, pp. 103-113, Jan. 1997.

7. K. Iagnemma, F. Genot, and S. Dubowsky, "Rapid physics-based rough terrain rover planning with sensor and control uncertainty," in IEEE ICRA 99, pp. 2286-2291, (Detroit, MI), May 1999.

8. B. D. Hoffman, E. T. Baumgartner, T. Huntsberger, and P. S. Schenker, "Improved rover state estimation in challenging terrain," Autonomous Robots 6(2), pp. 116-130, 1999.

9. P. Besl and N. McKay, "A method for registration of 3-d shapes," IEEE Transactions on Pattern Analysis and Machine Intelligence 14(2), pp. 239-256, 1992.

10. M. W. Walker, L. Shao, and R. A. Volz, "Estimating 3-d location parameters using dual number quaternions," CVGIP: Image Understanding 54(3), pp. 358-367, 1991. 